\title{
Weak CSS Sources from the FIRST Survey
}

\author{
Andrzej Marecki ${ }^{1}$, Jacek Niezgoda ${ }^{1}$, Jacek Włodarczak ${ }^{1}$, \\ Magdalena Kunert ${ }^{1}$, Ralph E. Spencer ${ }^{2}$ and Andrzej J. Kus ${ }^{1}$ \\ ${ }^{1}$ Toruń Centre for Astronomy, Nicholas Copernicus University, Toruń, Poland \\ ${ }^{2}$ Jodrell Bank Observatory, University of Manchester, UK \\ Received 2002 July 2, accepted 2002 November 23
}

\begin{abstract}
We report early results of an observational campaign targeted on a sample of compact steep spectrum sources selected from the FIRST survey which are significantly weaker than those investigated before. The selection criteria and procedure are given in detail. We present here an assortment of MERLIN and VLBI observations and make some general comments based on the morphologies of the sources presented.
\end{abstract}

Keywords: surveys — radio continuum: general — quasars: general — galaxies: active

\section{Sample Selection}

To select weak compact steep spectrum (CSS) sources from the FIRST catalogue (White et al. 1997) we took the following steps:

(a) From the source list based on Green Bank (GB) surveys at 21 and $6 \mathrm{~cm}$ (White \& Becker 1992) we selected those lying within the limits of the very first release of the FIRST survey - it covered the area RA $(\mathrm{J} 2000)=6^{\mathrm{h}} 35^{\mathrm{m}}-17^{\mathrm{h}} 30^{\mathrm{m}}, \operatorname{dec}(\mathrm{J} 2000)=28^{\circ}-42^{\circ}$ at that time - having steep spectra $\left(\alpha>0.5, S \propto v^{-\alpha}\right)$ and being stronger than $150 \mathrm{mJy}$ at $6 \mathrm{~cm}$. This flux density limit was chosen in order to produce a sample of a manageable size. The above declination limits indicate that the overlap between our sample and the B3-VLA survey-based sample (Fanti et al. 2001; Dallacasa et al. 2002a,b) is not large.

(b) Thanks to a dramatic difference in the resolution, the majority of sources appearing as single in the GB survey turn out to be double or multiple on FIRST maps. We rejected all such cases, i.e. we selected only those sources that are single entities in the FIRST catalogue, i.e. more compact than the FIRST beam (5".4) and surrounded by an empty field. We adopted 1 arcmin as a radius of that field. Additionally we referred to NVSS (Condon et al. 1998) to check that, indeed, we picked up compact sources. Such a procedure allows us to make sure that we deal with isolated objects and not parts of larger objects.

(c) We again checked whether our targets fulfill the spectrum steepness criterion: instead of GB survey flux densities at $21 \mathrm{~cm}$ we used more accurate values from FIRST. We rejected candidates with flat spectra $(\alpha \leq 0.5)$.

(d) We rejected the GPS sources. For this purpose we identified our preliminary candidates with objects listed in the $365 \mathrm{MHz}$ Texas catalogue (Douglas et al. 1996). We passed only those objects which have non-inverted spectra between 365 and $1400 \mathrm{MHz}$. In other words the turnover frequencies of our sources lie below $365 \mathrm{MHz}$.

Finally we selected 60 candidates for CSS sources.

\section{The Observations}

The initial survey was performed with MERLIN at $5 \mathrm{GHz}$. Each of our targets was observed six times in 10 min scans spread evenly over a $12 \mathrm{~h}$ track. Phase calibrators from the MERLIN Calibrator List were observed twice per target scan for 1-2 min.

Six objects having sizes similar to classical CSS sources, yet being less luminous, were followed up with MERLIN at $1.6 \mathrm{GHz}$ - see Kunert et al. (2002).

Twelve targets have either been misdetected or unresolved. The latter group of nine objects have just been followed up with the global VLBI. If it turns out that there are compact symmetric objects (CSOs) among them, this will be a very interesting result. Normally CSOs are GPS sources but our sources are not GPS by definition so they could be interpreted as 'dying CSOs'. We comment on this group in Marecki, Spencer, \& Kunert (2003, hereafter Paper II).

The whole set of MERLIN images is presented by A. Marecki et al. (in preparation). Twenty objects have been selected for higher resolution observations. Each of them was observed with the VLBA at $18 \mathrm{~cm}$ and the EVN at $6 \mathrm{~cm}$. Comprehensive results of those VLBI campaigns will be presented elsewhere. Here we draw some general conclusions based on the whole observational material and illustrate them with a small subset of images.

\section{Some General Conclusions}

1. Since our research was carried out at two frequencies and with three different interferometric arrays it enabled us to see our targets in several frequency/ resolution combinations. As a result we were able 

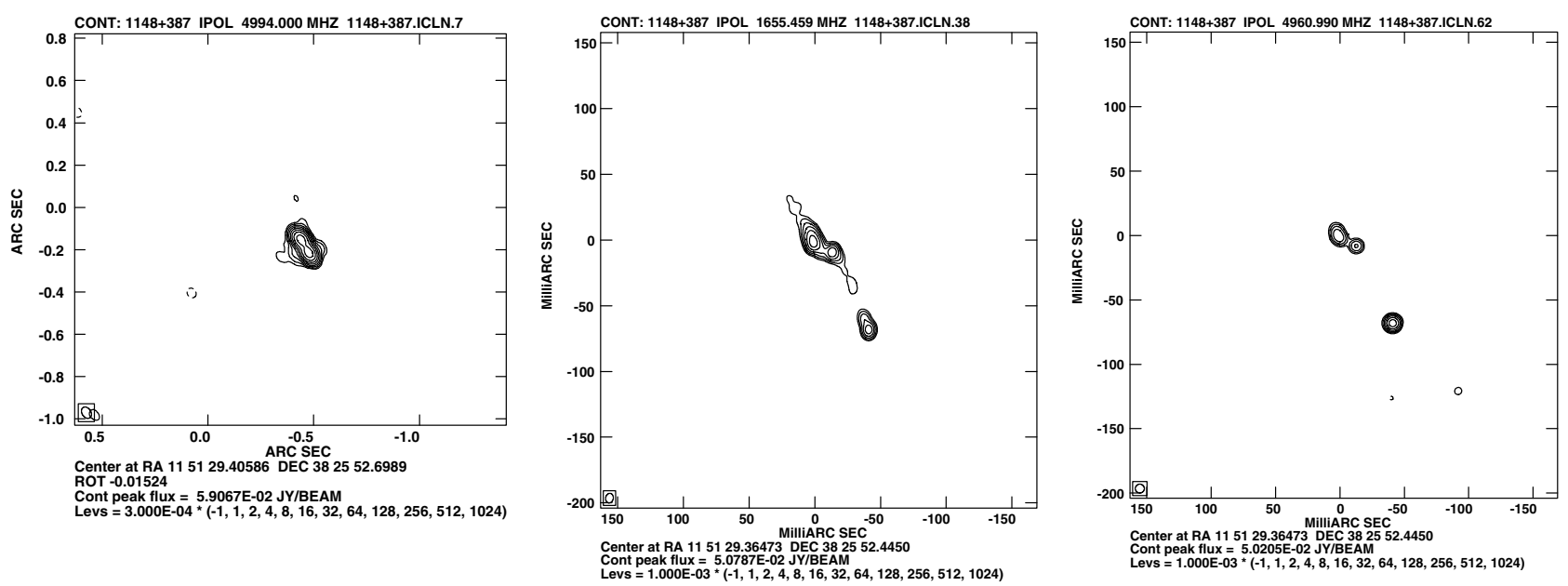

Figure 1 MERLIN $(6 \mathrm{~cm})$, VLBA $(18 \mathrm{~cm})$, and EVN $(6 \mathrm{~cm})$ maps of $1148+387$.
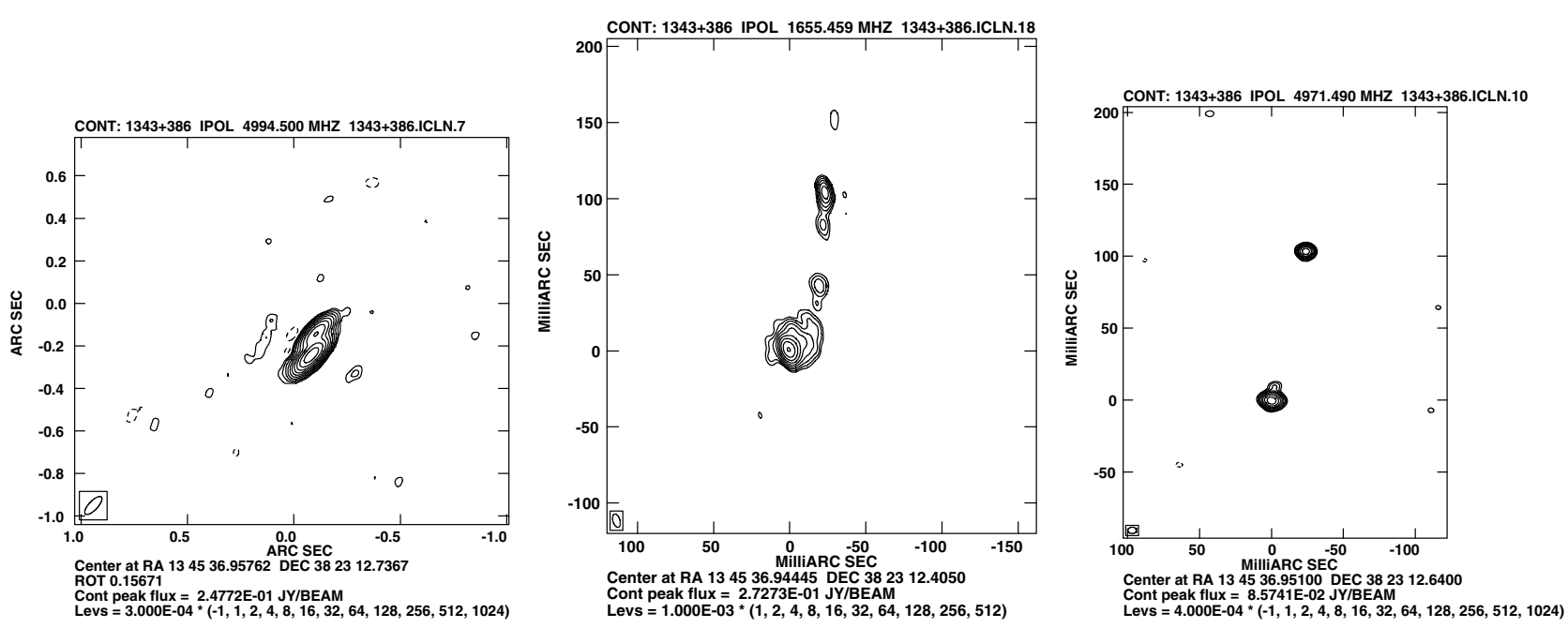

Figure 2 MERLIN $(6 \mathrm{~cm})$, VLBA $(18 \mathrm{~cm})$, and EVN $(6 \mathrm{~cm})$ maps of $1343+386$.

to locate cores in many objects and it appears that one-sided structures are not under-represented in our sample compared to other samples. In other words, it does not seem that CSS sources in deep samples like ours are predominantly symmetric objects. A single network, single frequency map can be very easily misinterpreted as an image of a symmetric object. The quasar $1148+387$ is a good example of such a deceiving object: it appears as a compact $(0.2$ separation of the components) and very symmetric double on the $6 \mathrm{~cm}$ MERLIN map but it is actually an asymmetric object according to our dual frequency VLBI surveys - the southern component has a flat spectrum so it must be a core (Figure 1). $1343+386$ (also a quasar), which was observed in parallel at $18 \mathrm{~cm}$ with the VLBA by Dallacasa et al. (2002a), is a similar case (Figure 2). According to our measurements, the component Dallacasa et al. (2002a) denoted as N1 is the strongest one and features a flat spectrum.

2. As in the case of other samples of CSS sources those which happen to be medium-sized symmetric objects
(MSOs) are FR II-like - 1709+303 is a classical specimen - and it is very hard to find a mini-FR I; in our sample only $1601+382$ resembles an FR I (Figure 3). We further comment on this fact in Paper II.

3. A classical FR II object (Fanaroff \& Riley 1974) is very clearly edge brightened thanks to the hot spots located at the source's extremities, and CSS sources, regardless of whether they are strong or weak, very often follow this pattern - see e.g. 1709+303. However, we found a few sources which, although in principle might be labelled FR II just because they are double-lobed but not FR Is, are not featured by well-defined hot spots. These are $1009+408,1236+327,1542+323,1656+391$, and $1717+315$ (Figures 4 and 5). Their lobes are diffuse and often 'amoeba-shaped' - 1542+323 (Figure 4) serves as the best example here. We think these are 'dying' sources, i.e. AGN where the central engine activity has stopped and the lobes are in the so-called 'coasting phase'. This subclass may be interpreted as a dead end of the evolution. We discuss this phenomenon in detail in Paper II. 

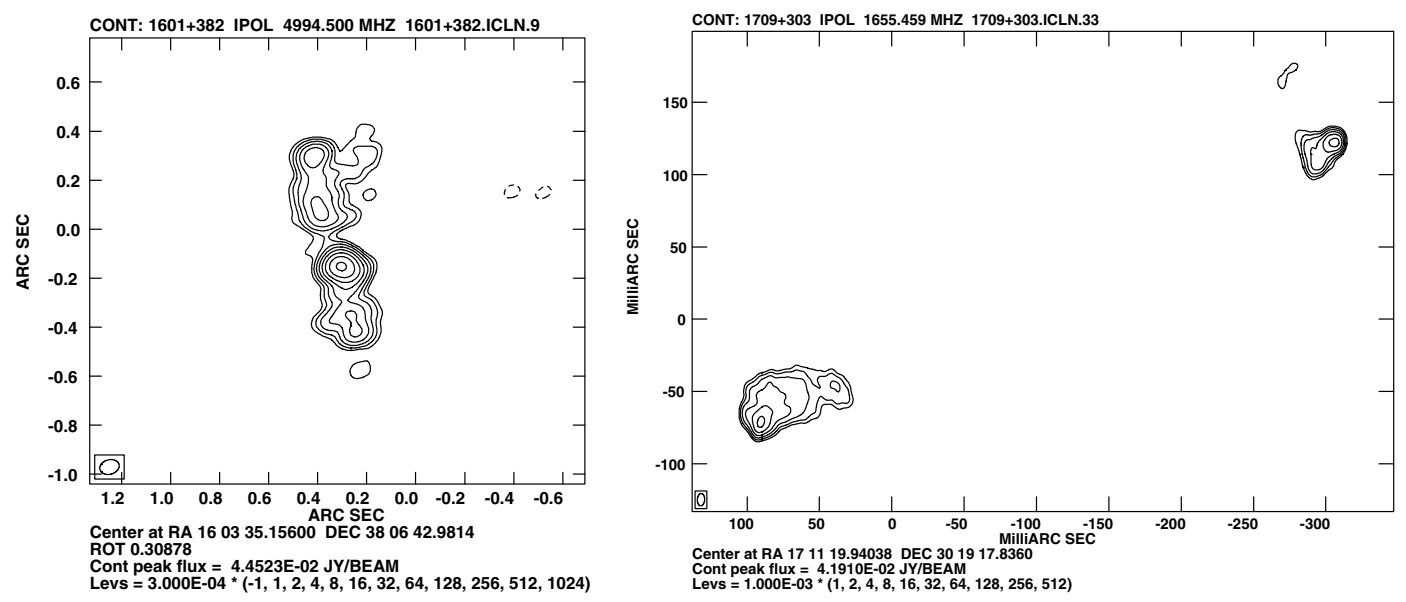

Figure 3 MERLIN (6 cm) map of 1601+382 (left); VLBA (18 cm) map of 1709+303 (right).
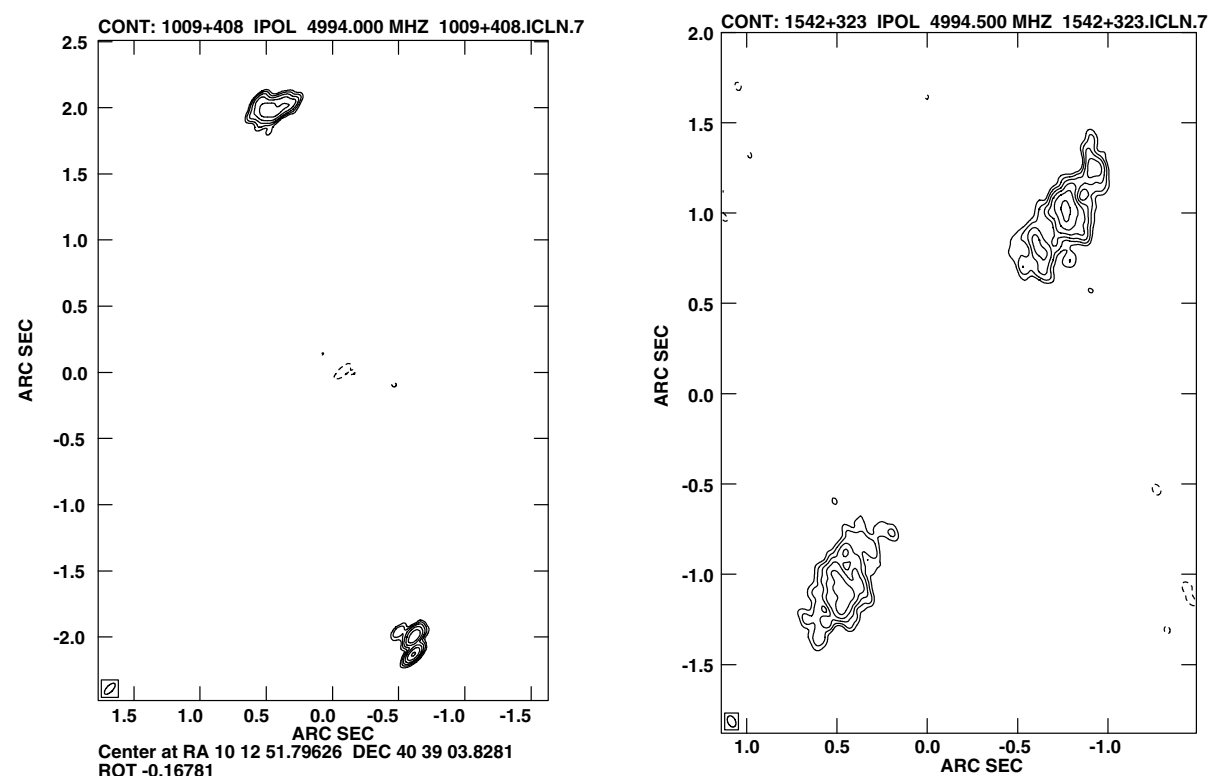

Cont peak fluX $=1.0508 E-02$ JY/BEAM

Cont peak $=3.000 \mathrm{E}-04 *(-1,1,2,4,8,16,32,64,128,256,512,1024)$
Len

Center at RA 154448.40281 DEC 320845.1691

ROT 0.27269

Cont peak flux $=1.0854 \mathrm{E}-02 \mathrm{JY} / \mathrm{BEAM}$

Levs $=3.000 \mathrm{E}-04 *(-1,1,2,4,8,16,32,64,128,256,512,1024)$

CONT: $1236+327$ IPOL $4994.000 \mathrm{MHZ} 1236+327$. ICLN.

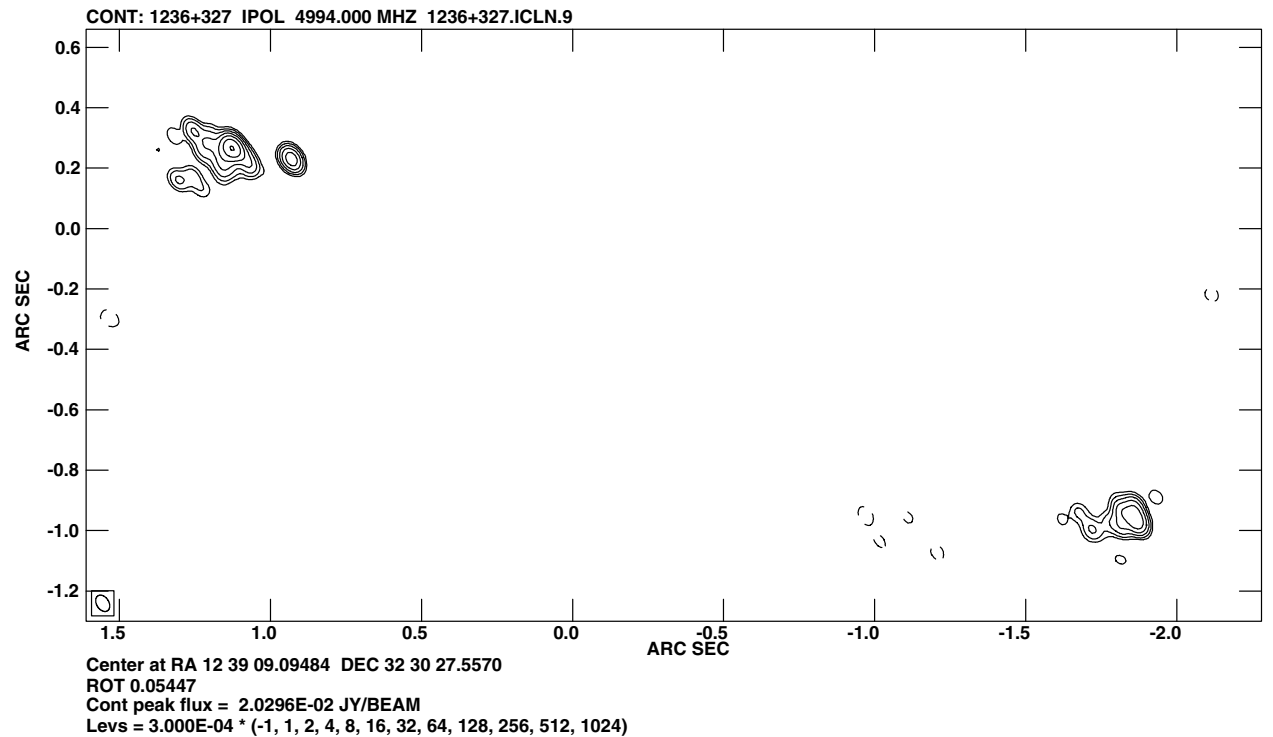

Figure 4 MERLIN (6 cm) maps of 'dying' arcsecond-scale CSS sources. 

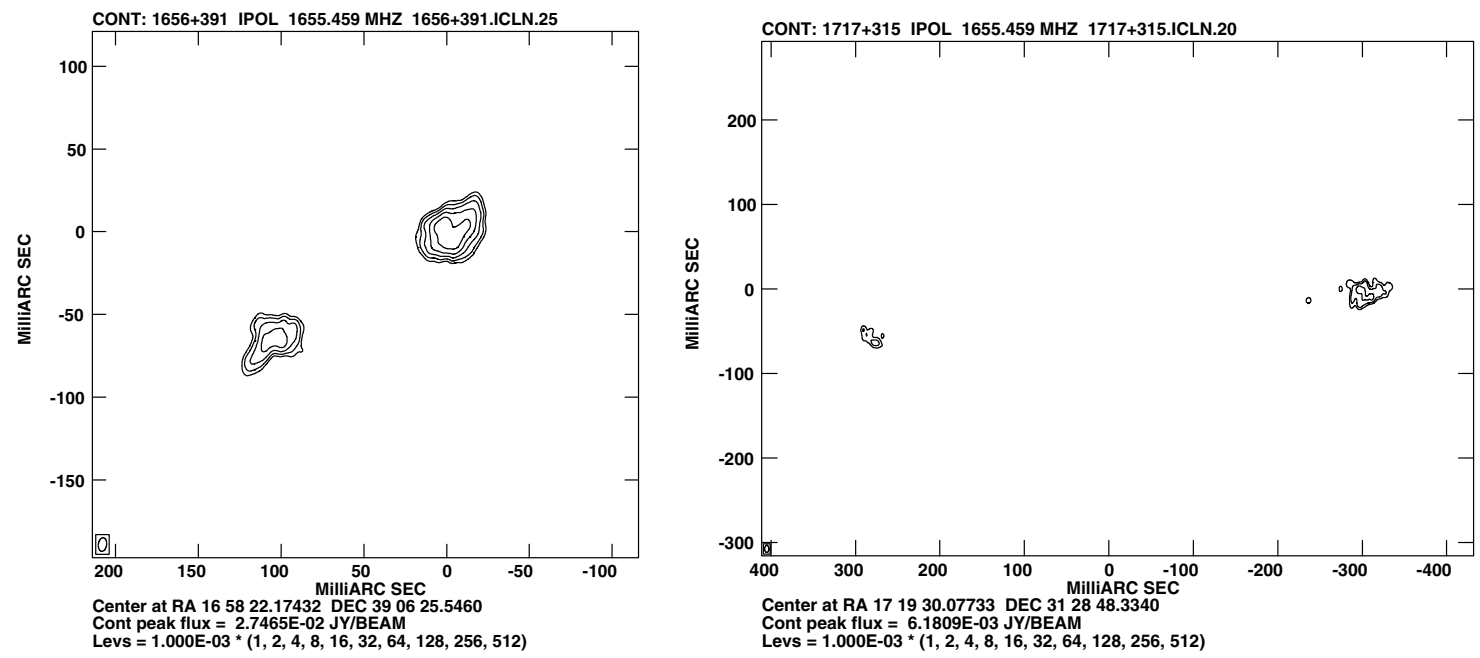

Figure 5 VLBA $(18 \mathrm{~cm})$ maps of 'dying' subarcsecond-scale CSS sources.

\section{References}

Condon, J. J., Cotton, W. D., Greisen, E. W., Yin, Q. F., Perley, R. A., Taylor, G. B., \& Broderick, J. J. 1998, AJ, 115, 1693

Dallacasa, D., Tinti, S., Fanti, C., Fanti, R., Gregorini, L., Stanghellini, C., \& Vigotti, M. 2002a, A\&A, 389, 115

Dallacasa, D., Fanti, C., Giacintucci S., Stanghellini, C., Fanti, R., Gregorini, L., \& Vigotti, M. 2002b, A\&A, 389, 126

Douglas, J. N., Bash, F.N., Arakel Bozyan, F., \& Torrence, G. W., 1996, AJ, 111, 1945
Fanaroff, B. L., \& Riley, J. M. 1974, MNRAS, 167, 31P

Fanti, C., Pozzi, F., Dallacasa, D., Fanti, R., Gregorini, L., Stanghellini, C., \& Vigotti, M. 2001, A\&A, 369, 380

Kunert, M., Marecki, A., Spencer, R. E., Kus, A. J., \& Niezgoda, J. 2002, A\&A, 391, 47

Marecki, A., Spencer, R. E., \& Kunert, M. 2003, PASA, 20, 46 (Paper II)

White, R. L., \& Becker, R. H. 1992, ApJS, 79, 331

White, R. L., Becker, R. H., Helfand, D. J., \& Gregg, M. D. 1997, ApJ, 475, 479 